\title{
Hypertension and Periodontitis: A Joint Report by the Italian Society of Hypertension (SIIA) and the Italian Society of Periodontology and Implantology (SIdP)
}

\author{
Rita Del Pinto ${ }^{1} \cdot$ Luca Landi $^{2}$. Guido Grassi ${ }^{3}$. Nicola Marco Sforza ${ }^{4}$. Francesco Cairo ${ }^{5}$. Filippo Citterio ${ }^{6}$. \\ Guerino Paolantoni ${ }^{7}$. Francesco D'Aiuto ${ }^{8}$. Claudio Ferri ${ }^{1}$. Annalisa Monaco ${ }^{9}$. Davide Pietropaoli ${ }^{9}$. \\ the Italian working group on Hypertension, Periodontitis (Hy-Per Group)
}

Received: 15 June 2021 / Accepted: 5 July 2021 / Published online: 25 September 2021

(c) The Author(s) 2021

\begin{abstract}
An accumulating body of evidence supports an independent association between high blood pressure (BP) and periodontitis, possibly mediated by low-grade inflammation. This joint report by the Italian Society of Hypertension (SIIA) and the Italian Society of Periodontology and Implantology (SIdP) working group on Hypertension and Periodontitis (Hy-Per Group) provides a review of the evidence on this topic encompassing epidemiology, biological plausibility, relevance, magnitude, and treatment management. Consensus recommendations are provided for health professionals on how to manage BP in individuals showing signs of poor oral health. In summary, (1) large epidemiological studies highlight that individuals with periodontal diseases have increased risk for high/uncontrolled BP independent of confounders; (2) mechanistically, low-grade inflammation might have a causal role in the association; (3) BP profile and control might benefit from periodontal treatment in pre-hypertensive and hypertensive individuals; (4) oral health status should be evaluated as a potential risk factor for high/ uncontrolled BP, and effective oral care should be included as an adjunct lifestyle measure during hypertension management. Further research is needed to optimize BP management in individuals with poor oral health.
\end{abstract}

Keywords Hypertension $\cdot$ Periodontitis $\cdot$ Cardiovascular diseases $\cdot$ Inflammation $\cdot$ Risk factors

\section{Introduction}

This article is co-published in the journals High Blood Pressure and Cardiovascular Prevention and Oral Diseases. https://doi. org/10.1007/s40292-021-00466-6 or https://doi.org/10.1111/odi. 14009.

\section{Luca Landi}

lulandi@fastwebnet.it

$\triangle$ Claudio Ferri

claudio.ferri@univaq.it

1 Unit of Internal Medicine and Nephrology, Department of Life, Health and Environmental Sciences, Center for Hypertension and Cardiovascular Prevention, San Salvatore Hospital, University of L'Aquila, L'Aquila, Italy

2 Private Practice, Via della Balduina, 114, 00136 Rome, Italy

3 Department of Medicine and Surgery, Clinica Medica, University of Milano-Bicocca, Milan, Italy

4 Private Practice, Bologna, Italy
Cardiovascular diseases (CVD) are the most common noncommunicable diseases globally and represent a leading cause of death worldwide, carrying noteworthy social and economic implications. Several modifiable conditions and

5 Research Unit in Periodontology and Periodontal Medicine, Department of Clinical and Experimental Medicine,

University of Florence, Florence, Italy

6 Department of Surgical Sciences, C.I.R. Dental School, University of Turin, Turin, Italy

7 Private Practice, Naples, Italy

8 Periodontology Unit, UCL Eastman Dental Institute and Hospital, University College London, London, UK

9 Unit of Oral Diseases, Department of Life, Health and Environmental Sciences, Prevention and Translational Research, Dental Clinic, San Salvatore Hospital, University of L'Aquila, L'Aquila, Italy 
unmodifiable traits contribute to the development of CVD, and high blood pressure, or hypertension, is among the major ones [1]. With the consistent and vast evidence from observational and intervention studies regarding the central role of inflammation in the pathogenesis of CVD, novel contributors to the cardiovascular risk profile are now emerging. Among others, periodontitis has been recently proposed as a modifiable non-traditional risk factor for CVD. Periodontitis is a chronic inflammatory non-communicable disease of the structures supporting the teeth, characterized by aberrant host immune fitness to the oral microbiome $[2,3]$.

Both hypertension and periodontitis are very common conditions, affecting at least one third of the adult population worldwide. Besides sharing common denominators, such as older age, smoking habits, male gender, overweight/obesity, low socioeconomic status, and poor education [4], novel evidence supports an independent association between the two. A recent mendelian randomization analysis has in fact provided the first proof of a common genetic background involving genes entailed in the immune function [5]. This evidence supports the pathogenic hypothesis of a proinflammatory milieu favoring both conditions, as well as related cardiovascular complications, in keeping with the mechanistic role for lowgrade systemic inflammation in the onset and progression of CVD.

Other professional societies have already acknowledged that adequate professional and home oral care are part of the non-pharmacological strategy for the management of cardiovascular risk [6]. In their recent consensus report in 2019, the World Heart Federation (WHF) and the European Federation of Periodontology (EFP) concluded that successful periodontal treatment influences CVD progression, and that active management of traditional cardiovascular risk factors, including hypertension, is required in the presence of periodontitis in high-risk patients and in those with established CVD [6]. Limited evidence was indeed available regarding the role of periodontal treatment in uncomplicated settings, including the low- and moderate-risk hypertensive patients as well as those with subclinical atherosclerotic CVD. Given the relevance of non-pharmacological strategies for cardiovascular prevention at any level of baseline risk, awareness is needed among professionals in the fields of oral and cardiovascular medicine with regards to preventive measures that can effectively and safely contribute to good global health and ultimately improve patient care.

Following their joint announcement [7], the Italian Society of Hypertension (SIIA) and the Italian Society of Periodontology and Implantology (SIdP) promoted a scientific board of experts (Hy-Per Group) with the aim of reviewing the published evidence on the association between hypertension and periodontitis and discussing the following topics:
(1) prevalence of hypertension in patients with periodontal diseases; (2) impact of periodontitis in treated hypertensive individuals; (3) potential causal links between these conditions; (4) incident hypertension in individuals with preexisting periodontitis; and (5) effect of periodontal treatment on blood pressure profile.

\section{Methods}

This joint report by SIIA and SIdP aims at summarizing the published evidence on epidemiological association, possible mechanistic links, and intervention studies of hypertension and periodontitis.

Panel members were asked to review the available evidence on BP and periodontal health (top-down approach) [8] and summarize the findings under five key questions [7]. Clinical and research implications for the medical and the dental Professionals were then summarized.

\section{Results}

\subsection{Do People with Periodontitis have a Higher Prevalence of Hypertension?}

The available literature on this topic is based on the case definition of periodontitis, for which several versions were developed over years. Herein we also report on association studies that examined the relation between active disease and BP.

\subsubsection{Case Definition of Periodontitis and Hypertension}

The case definitions of periodontitis in the assessment of the relation with $\mathrm{BP}$ were based on CAL, PPD or CPI according to literature [9-14].

3.1.1.1 Prevalence The prevalence of hypertension in the presence of periodontitis in individuals aged at least 16 years has been recently evaluated as a secondary outcome of a systematic review on the association between the two conditions using 30 prospective and retrospective studies published between 2003 and 2018 [15]. In 25 out of the 30 studies included in the analysis, the prevalence of hypertension was higher in individuals with a diagnosis of periodontitis (range 7-77\%) compared with those not suffering from the disease (range 4-70\%) [15], while one single study confirmed the same finding in men, but not in women [16]. In parallel, the prevalence of periodontitis was greater in 
individuals with hypertension (range 29-61\%) compared with those without this condition (range 17-39\%) [15]. The definitions of periodontitis and hypertension varied across studies.

3.1.1.2 Mean BP Values Observational evidence from 26 cross-sectional and case-control studies reporting on average mean systolic and diastolic BP in patients with $(\mathrm{N}=46034)$ and without $(\mathrm{N}=211309)$ periodontitis showed that the former had $4.5 \mathrm{mmHg}$ higher systolic BP (95\% C.I. 2.9-6.1 mmHg, $\mathrm{p}<0.0001)$ and $2.0 \mathrm{mmHg}$ higher diastolic BP $(95 \%$ C.I. $1.2-2.8 \mathrm{mmHg}, \mathrm{p}<0.0001)$ when compared with the latter [15]. Globally, the systolic BP component is more typically associated with periodontitis than the diastolic component.

3.1.1.3 Association Two recent meta-analyses have examined the association between periodontitis and arterial hypertension with concordant findings [15, 17]. Due to the use of several definitions in the included studies, both metaanalyses used adapted case definitions for periodontitis $[9,18]$. Hypertension was defined as $\mathrm{BP} \geq 140 \mathrm{mmHg} / 90$ $\mathrm{mmHg}$ or the use of antihypertensive medications, with additional non-confident definitions also for this condition $[15,17]$.

The association between moderate-severe periodontitis and hypertension spanned from 16 to $67 \%$ (16 studies included) [17] and from 22 to 53\% (20 studies included) [15], with evidence of a positive linear association driven by the severity of periodontitis. The reported findings should be interpreted in the light of the high heterogeneity observed $\left(\mathrm{I}^{2}>80 \%\right)$. In addition, despite multivariable adjustments were performed, specific subgroup analyses based on gender, age and race were lacking.

\subsubsection{Periodontal Disease Activity and Hypertension}

Studies assessing the relation between periodontal disease activity and hypertension are based on the measurement of gingival bleeding (BoP, \%), a marker of active periodontal inflammation.

An analysis of a multiethnic US representative sample of 6617 men and 7377 women aged $\geq 17$ years enrolled in the Third National Health and Nutrition Examination Survey (NHANES III) indicated that BoP was the only measure consistently and significantly associated with raised systolic BP after multivariate adjustments (age, sex, ethnicity CRP, creatinine, $\mathrm{Na}^{+} / \mathrm{K}^{+}$ratio, chronic conditions-including chronic heart diseases and/or stroke, emphysema and/ or asthma, arthritis and/or lupus, diabetes, thyroid disease, cancer, and goitre-, smoking, alcohol consumption, BMI, education and poverty-income ratio) [19]. Specifically, the authors found a linear effect of BoP on BP, with higher average systolic BP by $0.5(0.3,0.6) \mathrm{mmHg}$ and higher odds for diagnosis of hypertension ( $\mathrm{BP} \geq 140 \mathrm{mmHg} / 90 \mathrm{mmHg}$ ) (OR 1.1, 95\% CI 1.0-1.1, P <0.05) for every $10 \%$ increase in the extent of gingival bleeding [19].

The combination of gingival bleeding with markers of long standing disease (either PPD or CAL) in the periodontal inflamed surface area (PISA) was used to describe the association of poor periodontal health with BP in comparison with BoP alone among 8614 individuals (52.1\% women) aged $\geq 30$ years from NHANES III [20]. Participants were stratified according to PISA tertiles as "Not inflamed" $\left(\mathrm{PISA}=0 \mathrm{~mm}^{2}\right)$, "Moderately inflamed" $(0<$ PISA $<37.6$ $\mathrm{mm}^{2}$ ), and "Severely inflamed" (PISA $\geq 37.6 \mathrm{~mm}^{2}$ ). A double threshold for high/uncontrolled BP was used $(\geq 130 / 80$ $\mathrm{mmHg}$ [21] and $\geq 140 / 90 \mathrm{mmHg}$ [22]). The results indicate concordant findings for BoP and PISA in terms of their association with BP. In fact, compared with healthy periodontium, severe PISA and BoP were associated with $43 \%$ $(p<0.001)$ and $32 \%(p=0.006)$ higher odds of $B P \geq 130 / 80$ $\mathrm{mmHg}, 58 \%(\mathrm{p}<0.001)$ and $73 \%(\mathrm{p}<0.001)$ higher odds of $\mathrm{BP} \geq 140 / 90 \mathrm{mmHg}$, and with higher systolic BP by nearly $4(\mathrm{p}<0.001)$ and $5(\mathrm{p}<0.001) \mathrm{mmHg}$, respectively [20].

Another study investigated the association of bleeding gums with high/uncontrolled BP across the clinical spectrum of periodontal disorders (gingivitis; stable periodontitis, i.e. with $\mathrm{BoP}<10 \%$; unstable periodontitis, i.e. with $\mathrm{BoP} \geq 10 \%$ ) using data from 5396 NHANES III participants aged $\geq 30$ years [20]. Major confounders shared between hypertension and periodontal diseases (age, sex, ethnicity, poverty, BMI, hypertension diagnosis, use of antihypertensive medications, smoking habits, and diabetic status) were accounted for by applying survey-based propensity score matching (PSM). High/uncontrolled BP was defined as $\geq 130 / 80 \mathrm{mmHg}$ [21] and $\geq 140 / 90 \mathrm{mmHg}$ [22]. The results indicated that gingival bleeding contributes to increase the risk of high/uncontrolled BP, an effect that is amplified when BoP is considered in combination with chronic disease parameters. In particular, gingival bleeding (gingivitis; unstable periodontitis) was independently associated with $2.6 \mathrm{mmHg}$ higher systolic $\mathrm{BP}(\mathrm{p}<0.001)$ and with about $40 \%$ greater odds of high/uncontrolled BP (OR for BP $\geq 140 / 90 \mathrm{mmHg}: 1.38,95 \%$ CI $1.13-1.69, \mathrm{p}=0.002$; OR for $\mathrm{BP} \geq 130 / 80 \mathrm{mmHg}: 1.42$; 95\% CI $1.19-1.68$; $\mathrm{p}<0.001$ ) compared with no bleeding (healthy periodontium; stable periodontitis). Average systolic BP was higher among participants with unstable periodontitis than those with stable periodontitis $(+2.1 \mathrm{mmHg} ; \mathrm{p}<0.001)$ or gingivitis $(+5.3$ $\mathrm{mmHg} ; \mathrm{p}<0.001)$ [23]. 


\subsection{Do People Treating High Blood Pressure with Medications have a Higher Risk of Uncontrolled Hypertension in the Presence of Periodontitis?}

Evidence on the impact of periodontitis on the achievement of BP targets during antihypertensive treatment as the main study outcome is limited to only three cross-sectional studies, of which two on a multiethnic cohort of US individuals aged $\geq 30$ years reporting on hypertension control achievement in the presence of periodontal disease compared with the absence of the disease [24, 25], and the other involving a cohort of Puerto Rican elderly aged $\geq 70$ years reporting on the association with high BP in individuals with periodontal disease taking antihypertensive medications compared with those having normal BP values [26]. Globally, there is observational evidence that periodontitis is associated with increased risk of uncontrolled hypertension despite antihypertensive treatment.

\subsubsection{Cross-sectional Multiethnic Cohort of Treated Hypertensives $\geq 30$ Years}

The first study was conducted on hypertensive adults aged at least 30 years, of various racial/ethnic background (selfdeclared non-Hispanic Whites, non-Hispanic Blacks, Hispanics, and mixed race/ethnicity) enrolled in the 2009-2014 NHANES campaigns, who had at least one natural tooth and without a history of heart transplant, artificial heart valve, congenital heart disease except for mitral valve prolapse, and bacterial endocarditis [25]. The sample included a proportion of smokers, diabetics, obese, and dyslipidemic participants. BP was measured with a mercury sphygmomanometer according to standardized protocols, and the average of 3 consecutive BP readings taken on the same arm was used for analyses. Periodontal health status was assessed by dentists on a full-mouth, 6-sites periodontal examination in terms of PPD and CAL, and disease severity was based on the CDC/ AAP case definition for the disease [27]. The results indicate that individuals who were taking medications for high $\mathrm{BP}$ and suffered from periodontitis had $2-3 \mathrm{mmHg}$ higher mean systolic BP compared with those without the disease before and after controlling for relevant confounders, respectively (age, sex, ethnicity, BMI, smoking status; HbAlc, total and HDL cholesterol, triglycerides, creatinine, education, poverty level, and CRP). This was confirmed in both men and women and across all age ranges, from 30-44 years, to 45-64 years, to 65 years and above. A parallel assessment indicated that the difference in mean systolic BP based on the presence or absence of periodontitis could be even wider (about 3-7 mmHg) in individuals with diagnosed, but untreated hypertension from the same NHANES cohorts. If the most recent recommendations on hypertension management were to be applied to those individuals, the risk of failing to achieve BP values like those recommended by the 2017 ACC/AHA guidelines $(<130 / 80 \mathrm{mmHg}$ independent of age or comorbidities [28]) was $20 \%$ higher in the presence of periodontitis compared with the absence of the disease (OR 1.20, 95\% CI 1.05-1.37, p=0.008). Of note, the finding remained significant after progressive adjustment for the above mentioned possible confounders, except when serum CRP was included in the model (OR 1.19, 95\% CI $0.91-1.54, p=0.2$ ), suggesting a possible mediating effect of systemic inflammation in the observed association, although fluctuations in the inflammatory response during chronic periodontitis with impact on serum CRP levels should be considered as an alternative explanation.

The same sample was re-analyzed based on the redefinition of hypertension treatment goals according to 2018 European Society of Cardiology/European Society of Hypertension (ESC/ESH) Guidelines for the management of arterial hypertension, which are modeled according to patients characteristics $(<130 / 80 \mathrm{mmHg}$ if aged $<65$ years and with normal renal function, $<140 / 80 \mathrm{mmHg}$ if aged $\geq 65$ years or with chronic kidney disease, independent of age [22]) [24]. The full-adjusted (sex, ethnicity, BMI, smoking habits, glycohemoglobin, cholesterol, triglycerides, education, poverty level, CRP) risk of uncontrolled hypertension was $36 \%$ higher in the presence of periodontitis compared with the absence of the disease when using the $2018 \mathrm{ESC} / \mathrm{ESH}$ treatment goals as reference (OR 1.36, 95\% CI 1.05-1.78, $\mathrm{p}=0.02)[24]$.

\subsubsection{Cross-sectional Hispanic Cohort of Treated Hypertensives $\geq 70$ Years}

The second study was on 185 elderly individuals (62 males, 123 females, age range 70 to 97 years) with preserved cognitive function from the Puerto Rican Elderly Dental Health Study (PREDHS), an ancillary cohort of the Puerto Rican Elderly: Health Conditions (PREHCO) study [26]. The sample included smokers, diabetic, and obese individuals. Major reasons for exclusion from PREDHS were use of anticoagulants, renal dialysis, heart valve problems, congenital heart disease, endocarditis, hip bone or joint replacement, rheumatic fever, hemophilia, having pacemaker or defibrillator or any cardiovascular prosthesis. BP was measured on the right arm using a bell stethoscope and a mercury sphygmomanometer according to standard procedures, and antihypertensive medications were identified by a cardiologist blinded to periodontal status and BP values of participants. High BP was defined as average systolic $\geq 140 \mathrm{mmHg}$ or average diastolic $\geq 90 \mathrm{mmHg}$. Periodontal health status was assessed on a full-mouth, 4-sites periodontal examination performed during home visits by 3 teams of calibrated dental examiners, and it was expressed in terms of PPD and CAL. 
Disease severity was based on the CDC/AAP case definition for the disease [11]. A multivariate logistic regression was performed to evaluate the associations between periodontal disease and high BP, and a subgroup analysis was performed comparing participants taking antihypertensive medications with those having normal BP values. After controlling for potential confounders including age, sex, smoking and drinking habits, diabetes, level of physical activity in the past month, BMI, dietary habits, and home/professional dental care, severe periodontal disease was associated with more than a four-fold increase in the risk of high BP among individuals taking antihypertensive medications compared with individuals with normal BP (OR 4.63, 95\% CI 1.20-17.94). The authors did not assess the risk of high BP in treated hypertensive individuals with periodontitis compared with those with a healthy periodontium.

\subsection{Is There Any Evidence for a Causal Link Between Periodontitis and Hypertension?}

Herein, the plausible genetic bases linking periodontitis and hypertension are described. The possible trigger role of environmental factors, like high-salt diet, and local dysbiosis is also reported.

\subsubsection{Heritability of Periodontitis and Hypertension}

While monogenic hypertension depends on defects of specific genes mainly involved in sodium handling and steroid hormone metabolism [29], the most common form of hypertension is a complex trait grounded on a polygenic basis and favored by environmental factors, where potentially inheritable epigenetic modifications concur to the clinical phenotype [30]. Alternative hypotheses to explain the pathophysiology of hypertension that are not centered on the kidney have recently found confirmation in an association study on 128,272 SNPs from 342,415 individuals of European ancestry. The authors identified 66 genome-wide significant loci for BP, of which 17 were novel, that cumulatively explained nearly $3.5 \%$ of the trait, with broadly comparable BP effects in South Asians, East Asian and Africans [31]. These variants were enriched for cis-regulatory elements particularly in vascular endothelial cells, suggesting their possibly relevant role in BP control through modulation of vascular tone at the systemic level. The study provided a consistent genetic basis for increased systemic vascular resistance as a pathogenic event in the development of hypertension that possibly involves endothelial permeability or vascular smooth muscle cell contractility, arguing against a single dominant renal effect in the genetic determination of intra-arterial pressure. In a study on a series of 229 monozygotic and dizygotic twins, CRP secretion itself was described as to be substantially heritable in humans, sharing genetic determination with metabolic features including BP [32]. Specifically, significant associations were found between CRP concentration and polymorphisms in the catecholaminergic/b-adrenergic pathway, providing a biological proof of concept for the role and actions of inflammation within the adrenergic system and the clinical hypertensive phenotype [32].

A certain degree of heritability was also described for periodontitis, ranging from $15 \%$ in family studies $\left(\mathrm{H}^{2} 0.15\right.$, 95\% CI $\left.0.06-0.24 ; \mathrm{I}^{2}=0 \%\right)$ to $38 \%$ in twin studies $\left(\mathrm{H}^{2} 0.38\right.$, $95 \%$ CI $0.34-0.43 ; \mathrm{I}^{2}=12.9 \%$ ), with higher heritability for severe early-onset traits and younger individuals [33]. When studies were combined, summing up to more than 50,000 participants, nearly a third of the periodontitis variance in the population was attributable to genetic factors $\left(\mathrm{H}^{2} 0.29\right.$, 95\% CI $0.21-0.38 ; \mathrm{I}^{2}=61.2 \%$ ) [33]. Several suggestive susceptibility loci for periodontitis have been reported by genome-wide association studies (GWAS) [34, 35], some of which are intertwined with the immune system [36]. This is the case for a locus at SIGLEC5 (sialic acid binding Ig-like lectin 5) and a chromosomal region downstream of the DEFA1A3 locus (defensin alpha 1-3), which showed association with both chronic and aggressive disease and were associated with periodontitis at a genome-wide significance level [36]. The DEFA genes belong to the family of alpha defensins that are believed to be involved in phagocyte-mediated host defense against bacteria, fungi and viruses, and there is evidence of their strong expression in neutrophils and macrophages, as well as in the epithelia of mucosal surfaces. SIGLEC5 (CD170) belongs to the human CD33-related siglecs and is broadly expressed in various myeloid cells of the innate immune system and in B cells. It seems to function as an inhibitory receptor for myeloid cells to prevent inappropriate reactivity against self-tissues $[37,38]$. Accordingly, it appears to be involved in maintaining leukocytes in the quiescent state until proper activation occurs through specific receptors [38].

\subsubsection{Evidence for a Genetic Association Between Periodontitis and Hypertension}

A two-sample Mendelian randomization analysis has been recently performed on the nearly 750,000 UK-Biobank/ International Consortium of Blood Pressure-Genome-Wide Association Studies participants to ascertain the effect on BP of 4 SNPs already associated with periodontitis in GWAS studies [5]. The examined polymorphisms included those in DEFA1A3 (rs2738058) and SIGLEC5 (rs4284742) loci, which are involved in the immune function. All four SNPs were considered as a periodontitis exposure proxy and were tested in the context of the BP phenotypes. GWAS data on $\mathrm{BP}$ were used to extract estimates of the association between the 4 SNPs and systolic and diastolic BP [39]. The results showed that, for all the examined SNPs, the same allele was 
associated with both increased risk of periodontitis and increased levels of BP [5]. Thus, this pioneer study provided the first evidence of a causal, genetic-grounded relationship between periodontitis and BP that involved, at least in part, the immune system.

\subsubsection{High-Salt Diet and Dysbiosis}

A role for immune cells, like regulatory $\mathrm{T}$ cells (Tregs), $\mathrm{T}_{\mathrm{H}} 17$, and neutrophils, has been described for both periodontitis and hypertension [4]. Environmental factors, like high-salt diet [40, 41], as well as an altered microbiome [42] were shown to act as possible triggers to immune cells impairment ( $\mathrm{T}_{\mathrm{H}} 17$, neutrophils) with effects on periodontal health status and BP. Specifically, a high-salt diet, which is a common environmental trigger for hypertension, taken for 1 week was shown to impair antibacterial responses of neutrophils through salt-induced hyperglucocorticoidism and consequent glucocorticoid-mediated immunosuppression [41]. High-salt diet was also found to trigger tissue inflammation via $\mathrm{T}_{\mathrm{H}} 17$ cell development mediated by an increase in serum glucocorticoid kinase 1 (SGK1) expression [40]. In parallel, a dysbiotic microbiome in the oral cavity was found to trigger $\mathrm{T}_{\mathrm{H}} 17$ cell expansion, which mediated inflammatory tissue destruction in experimental periodontitis [42].

\subsection{Are People with Periodontitis More Likely to Develop Hypertension in the Future?}

Longitudinal evidence assessing the incidence of hypertension in relation with periodontal diseases is limited, including only two studies based on self-reported data, of which one on middle aged and old adult men [43] and the other on post-menopausal women [44], and two studies where clinical $\mathrm{BP}$ values and periodontal health status markers were measured, conducted on young adults and adults of both sexes $[45,46]$. In contrast with the first two studies, where no association between self-reported periodontal health status and BP was found, an increased risk of incident hypertension in relation to periodontal disease was found in the studies based on BP values and oral health status assessments.

\subsubsection{Cohorts with Self-reported BP and Periodontal Information}

The first is a study on a prospective cohort of male health professionals (dentists, pharmacists, optometrists, podiatrists, osteopaths and veterinarians) enrolled in The Health Professionals' Follow-Up Study (HPFS) in 1986 who selfreported individual data on lifestyle and medical conditions on a biennial basis throughout the 20-years follow-up period (median follow-up: 16.3 years), in the assumption that their professional education and high socioeconomic status granted for data accuracy [43]. Participants were aged 40-75 years at enrollment. The study lacks measured data on periodontal health status as well as on actual BP values. Diagnosed periodontitis (yes/no) was asked at baseline and every two years thereafter, and the severity of periodontal bone loss (none, mild, moderate, severe) was investigated with a question in 1996. The examined sample consisted of 31,543 eligible participants $(466,514$ person-years during 20 years of follow-up). After adjusting for potential selfreported confounders (family history of hypertension, diabetes diagnosis, alcohol consumption, smoking habits, physical activity, BMI, use of supplemental multivitamins or vitamins $\mathrm{E}$ or D or calcium, fruit/vegetable intake, cumulative tooth loss, and periodontal disease diagnosis during the follow-up), no association was observed between self-reported baseline periodontal disease and incident hypertension (relative risk $[\mathrm{RR}]=1.04$; 95\% CI: 0.98-1.10), nor between selfreported periodontitis during follow-up $(\mathrm{RR}=1.01 ; 95 \% \mathrm{CI}$ : 0.96-1.05), tooth loss during follow-up ( $\mathrm{RR}=1.03$; $95 \% \mathrm{CI}$ : 0.98-1.09), severe periodontal bone loss $(\mathrm{RR}=1.02 ; 95 \%$ CI: 0.77-1.35), or number of teeth reported at baseline $(<10$ or $\geq 25$ teeth $)(\mathrm{RR}=1.05 ; 95 \% \mathrm{CI}: 0.91-1.21)$ and the same outcome [43].

The association of baseline self-reported periodontal disease and edentulism with incident hypertension in women was assessed within the Women's Health InitiativeObservational Study (WHI-OS) [44]. It was a prospective cohort study of 93,676 postmenopausal women aged 50-79 years at the time of enrollment between 1993 and 1998 and followed-up through 2015 (mean follow-up 8.3 years). Only participants who self-reported information on their periodontal health status and number of teeth at the 5-year questionnaire post-enrollment (1998-2003) and concomitantly self-reported neither being diagnosed with hypertension nor taking antihypertensive medications were included $(\mathrm{N}=36,692)$. Information on the severity or activity of self-reported periodontal disease was not available. After accounting for potential confounders (age, ethnicity, smoking status, BMI, alcohol intake, total physical activity, diabetes, frequency of dental visits, baseline systolic BP measured with auscultatory method using a conventional sphygmomanometer, and dietary factors including consumption of red meat, sodium, and total calorie intake), women with self-reported edentulism had approximately $20 \%$ higher risk of incident hypertension compared with dentate women (HR 1.21, 95\% CI 1.11-1.30). Conversely, self-reported periodontal disease was not associated with incident hypertension (HR 0.99, 95\% CI 0.95-1.03).

\subsubsection{Cohorts with Measured BP and Periodontal Data}

The first study enrolled a sample of Japanese employees (727 males, 296 females, mean age 37.3 years, age range 
20-56 years) [46], who underwent medical and dental checkups in 2002 and after 4 years and in whom BP and the other metabolic syndrome components were within normal ranges at baseline. BP was measured with an automatic device in a sitting position and a single measurement was performed (two in case an abnormal value at the first measurement was recorded). Hypertension was defined as systolic $\mathrm{BP} \geq 130-\mathrm{mm} / \mathrm{Hg}$ or diastolic $\mathrm{BP} \geq 85-\mathrm{mm} / \mathrm{Hg}$ based on the threshold for the definition of metabolic syndrome in Japan. Periodontal health status was assessed by dental hygienists supervised by dentists with standard protocols in terms of periodontal pockets (PPD $\geq 4 \mathrm{~mm}$ ). After 3 years, $13.7 \%$ of individuals developed hypertension. After adjustments for age, sex, tobacco smoking, exercise, eating habits, and BMI, the risk of incident hypertension was significantly associated with the baseline presence of periodontal pockets (OR $1.5,95 \%$ CI 1.0-2.3, p < 0.05) [46]. Conversely, one or more missing teeth at baseline were not associated with incident hypertension.

The second study included a sample of Japanese university students $<30$ years (1278 males, 1310 females, mean age 18.2 \pm 0.7 years, age range $18-27$ years) with no selfreported history of treated/untreated hypertension, who underwent baseline general health and oral examinations in April 2010-2011 ( $=3011)$ and 3-year follow-up examination in April 2013-2014 ( $\mathrm{N}=2588$; follow-up rate: 66.7\%) [45]. BP and heart rate were obtained by using an automatic oscillometric device and BP was categorized based on the measured BP values (normal BP: $<120 / 80 \mathrm{mmHg}$;

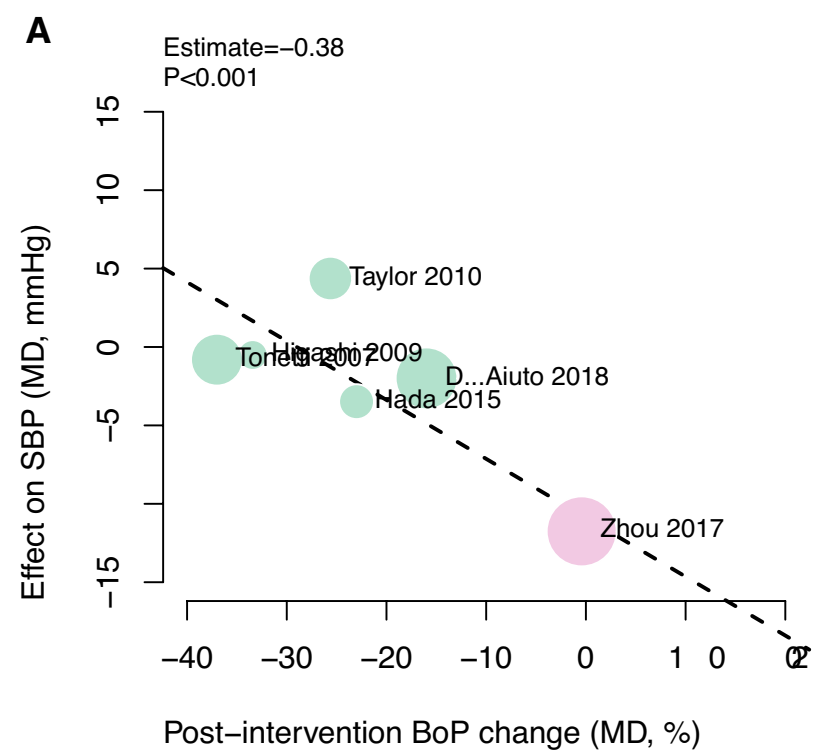

Fig. 1 Effect of periodontal treatment on systolic BP in pre-hypertensive and hypertensive individuals. A meta-regression between post-intervention BoP change (mean difference [MD]; \%) and systolic $\mathrm{BP}(\mathrm{MD}, \mathrm{mmHg})$. B meta-regression between post-intervention $\mathrm{PPD}$ change $(\mathrm{MD} ; \mathrm{mm})$ and systolic $\mathrm{BP}(\mathrm{MD}, \mathrm{mmHg})$. Regression prehypertension: systolic BP $120-139 \mathrm{mmHg}$ or diastolic BP $80-89 \mathrm{mmHg}$; hypertension: $\geq 140 / 90 \mathrm{~mm} \mathrm{Hg}$ ). A second or third measure was taken if BP values were $>140 / 90$ $\mathrm{mm} \mathrm{Hg}$; otherwise, only one BP measurement was recorded. Periodontal health status was assessed by 6 calibrated dentists with standard protocols in terms of teeth count, PPD, BoP, as well as simplified oral hygiene index for dental plaque and calculus. BMI was based on measured weight and height. Gender, age, general health conditions, eating, drinking and smoking habits, physical activity, as well as home and professional oral care habits were self-reported. There were very few current smokers and no former smokers in the enrolled sample. Based on multivariate logistic regression accounting for age, gender, smoking status, BMI, eating habits, number of teeth, simplified oral hygiene index, and baseline periodontal disease, the predictors of developing hypertension at the 3-year follow-up among individuals with baseline prehypertension included male sex (OR: 6.31; 95\% CI: 2.63-15.13; $\mathrm{p}<0.001)$, baseline sedentary lifestyle (OR: 2.90; 95\% CI: 1.56-5.38; $\mathrm{p}<0.01$ ), and baseline periodontal disease defined as the presence of PPD $\geq 4 \mathrm{~mm}$ and BOP $\geq 30 \%$ (OR: 2.74; 95\% CI: 1.19-6.29; $\mathrm{p}=0.02$ ). Conversely, baseline periodontal disease did not predict the risk of developing prehypertension (OR: $0.93 ; 95 \% \mathrm{CI}$ : $0.51-1.70 ; \mathrm{p}=0.82$ ).

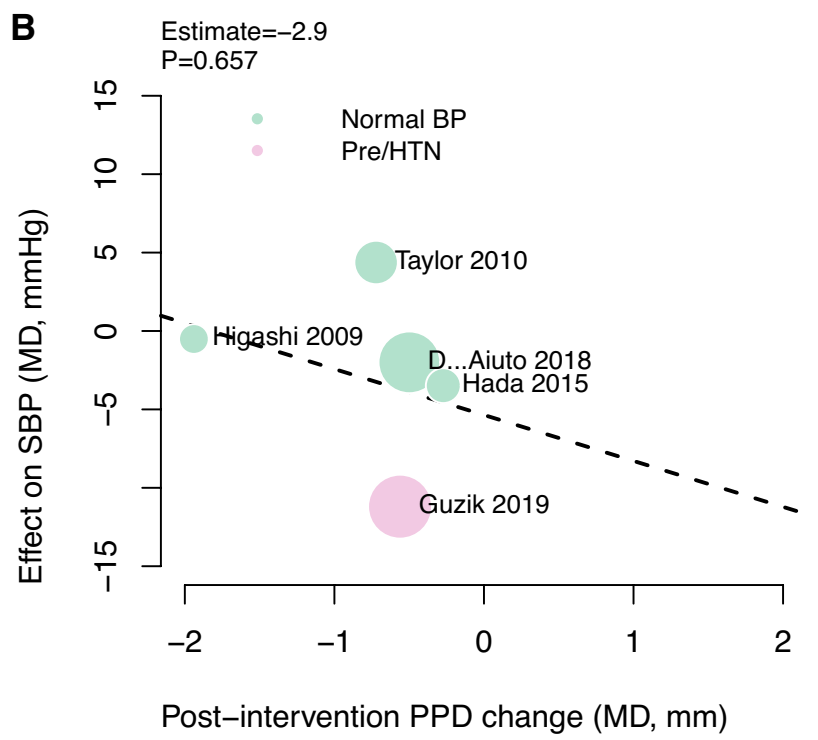

slopes reflect the effect of periodontal treatment on BP in pre-hypertensive and hypertensive individuals. Data were extracted from studies included in the meta analysis by Sharma et al. [47] and the metaregression is an original figure by the Hyper-Group. Heterogeneity was reported among studies 


\subsection{Is There an Effect of Periodontitis Treatment in Lowering BP and Improving BP Control in Hypertensive People?}

In this section, the effect of periodontal therapy on BP as the primary outcome in three studies on individuals with prehypertension, treated hypertension, or resistant hypertension is examined. Globally, the results show a parallel improvement in systemic inflammatory markers and BP profile after treatment (Fig. 1). One study assessed as a secondary outcome the effect of nonsurgical periodontal therapy on BP in treated hypertensive individuals, reporting no significant changes. The impact of periodontal treatment on BP in normotensive individuals has been recently addressed [47] with evidence of no benefits (Fig. 1) and will not be discussed here.

\subsubsection{Effect of Periodontal Therapy on Blood Pressure as the Primary Outcome}

To date, the effect of periodontal therapy on blood pressure as the primary outcome has been reported by three studies $[5,48,49]$. Two studies were randomized clinical trials, one of which involved pre-hypertensive individuals (systolic BP between 120 and $139 \mathrm{mmHg}$ or diastolic BP between 80 and $89 \mathrm{mmHg}$ according to the Seventh Report of the Joint National Committee on Prevention, Detection, Evaluation, and Treatment of High Blood Pressure [50] and assessed the outcome in terms of clinical BP [48], and the other one [5] included hypertensive individuals on a stable antihypertensive therapy since at least 6 months prior to enrollment and assessed the outcome using 24-hours ambulatory BP measurement (ABPM) [22]. Both RCTs involved participants with moderate to severe periodontitis [11, 27] who were randomized to either conventional or intensive periodontal treatment.

The third study [49] was a prospective cohort pilot study on individuals $\geq 40$ years with severe essential refractory hypertension for at least 2 years of good adherence on a guidelines-based antihypertensive treatment strategy, and with generalized advanced chronic periodontitis. BP was assessed using an ABPM device [51].

3.5.1.1 Pre-hypertension The pre-hypertensive population trial [48] assigned patients in the intensive-treatment group to full-mouth intensive removal of dental plaque biofilms with the use of scaling and root planing, teeth extraction as needed, and local antibiotic delivery (minocycline) into the periodontal pockets once weekly for 4 consecutive weeks. Patients in the control-treatment group only accepted a conventional cycle of supragingival scaling and polishing at baseline. In addition, all patients received basic oral hygiene instructions by experienced periodontists. The total length of follow-up was 6 months, with intermediate assessments at 1 and 3 months. Clinical BP was measured using an automated device in a controlled setting [52], and the average of a minimum of two measurements recorded 2 minutes apart was used in the study.

Individuals in the intensive-treatment group $(\mathrm{N}=53)$ compared with those in the conventional-treatment group $(\mathrm{N}=54)$ showed a larger improvement in clinical measures of periodontitis (PPD, CAL, BoP) at 3 and 6 months. In the former, but not in the latter, this change was paralleled in the same time points by a decrease in clinical systolic and diastolic BP, systemic inflammation expressed as hsCRP, and circulating markers of endothelial function. The betweengroups difference in systolic BP was visible as early as the first month follow-up visit, while that in IL-6 emerged at the 6 months follow-up visit only.

Specifically, systolic BP in the intensive-treatment group decreased from $129.31 \pm 5.85 \mathrm{mmHg}$ (baseline) to $125.83 \pm 5.30 \mathrm{mmHg}$ ( 1 month follow-up visit), $122.17 \pm 5.15 \mathrm{mmHg}$ (3 months follow-up visit), and $118.23 \pm 5.45 \mathrm{mmHg}$ (6 months follow-up visit). The diastolic BP component also decreased progressively from baseline to study end in this group. Conversely, neither systolic or diastolic BP decreased in the conventional-treatment group. The absolute between-groups difference in BP at 6 months was $12.57 \mathrm{mmHg}$ for systolic BP $(\mathrm{p}<0.001)$ and $9.65 \mathrm{mmHg}$ for diastolic BP $(\mathrm{p}<0.001)$. The observed change in clinical BP in the intensive-treatment group was accompanied by a decrease in hs-CRP, IL-6 and circulating markers of endothelial function (CD31+/CD42- endothelial microparticles), which did not occur in the conventionaltreatment group, resulting in significant between-groups differences in all parameters at the study end [48].

3.5.1.2 Treated Hypertension This RCT enrolled individuals with treated essential hypertension, with or without other cardiovascular risk factors such as overweight, tobacco smoking, type 2 diabetes mellitus, and hypercholesterolemia [5]. Changes to antihypertensive medications during trial resulted in the exclusion from analysis. Intensive periodontal treatment consisted of a single session of full-mouth supragingival and subgingival tooth scaling, while conventional treatment consisted of a single session of supragingival tooth scaling. Any additional required periodontal treatment in individuals randomized to this group was postponed to the study end. All participants received dental hygiene instructions. Ambulatory BP was assessed using Spacelabs Ultralite 90217 devices in accordance with manufacturer recommendations and in agreement with the latest available protocols [22]. Full dental examination and cardiovascular evaluation, comprehensive of ABPM, blood samples collection for flow cytometry analysis and plasma cytokine measurement, as well as vascular function assess- 
ment by flow-mediated dilatation (FMD) of the brachial artery, were repeated 2 months after periodontal therapy.

Intensive periodontal treatment $(\mathrm{N}=45$ patients) produced a greater benefit in terms of periodontal health compared with control treatment ( $\mathrm{N}=46$ patients), leading to a significant reduction in the examined clinical measures of periodontitis (PPD, CAL). Conventional treatment determined only a small reduction in PPD. Changes in periodontal health parameters were paralleled by a decrease in ambulatory $24 \mathrm{~h}$ average systolic and diastolic BP only in the intensive-treatment group, leading to an absolute betweengroups difference in systolic and diastolic BP of $11.1 \mathrm{mmHg}$ (95\% CI 6.5-15.8) and $8.3 \mathrm{mmHg}(95 \%$ CI 3.98-12.6), respectively. The benefit of intensive treatment on BP was visible on both the daytime and nighttime BP profiles, and it was not affected by the number of antihypertensive medications ( $>2$ or $\leq 2)$. Conversely, it depended on baseline BP and age, with no significant changes in systolic BP among individuals with ambulatory 24 hours average systolic BP in the highest tertile ( $>138 \mathrm{mmHg}$ ), and larger benefit in young individuals (age $<58$ years). A formal mediation analysis indicated a significant average causal mediation effect of treatment group on systolic BP reduction attributable to changes in PPD [estimate $=3.59$ (95\% CI 0.52-7.10), $\mathrm{p}=0.021$; proportion of total mediated effect $=0.29(95 \%$ CI 0.04-0.61), $\mathrm{p}=0.021$ ]. Intensive, but not control periodontal treatment modestly yet significantly reduced IFN $\gamma$ and IL-6 levels as well as the levels of activated (CD38+) and immunosenescent (CD28null, CD57+) proinflammatory CD8+T cells, which have been shown to infiltrate kidneys and vessels in the early phase of vascular injury in hypertensive patients.

Heart rate was not modified by periodontal treatment.

3.5.1.3 Resistant Hypertension In the interventional prospective cohort pilot study on adults with severe essential refractory hypertension $(\mathrm{N}=26$; mean age: $53.6 \pm 8.0$ years; mean BMI: $30.6 \pm 8.0 ; 65.4 \%$ women; $61.5 \%$ non-Whites; $11.5 \%$ smokers; $15.4 \%$ diabetics; $23.1 \%$ with a history of myocardial infarction or stroke), periodontal examination, cardiological assessment (ABPM, echocardiography for the assessment of left ventricular mass, PWV) and laboratory tests on plasma inflammatory markers were undertaken at baseline and 3 and 9 months thereafter [49]. All participants suffered from generalized advanced chronic periodontitis [10] diagnosed on a complete periodontal examination performed by the same calibrated periodontist. Non-surgical periodontal treatment was delivered as needed by a single experienced periodontist 3 months after the baseline visit. Treatment consisted of supragingival scaling using manual instruments and a sonic device, and subgingival scaling and root planing in sites with PPD $\geq 4 \mathrm{~mm}$, with no limit of appointments and an average of four to six visits per patient within a 2-week period. All participants also received reiterated oral hygiene instructions consisting of a demonstration of dental floss use and Bass technique for brushing. Periodontal treatment determined a significant improvement in clinical parameters of the disease (BoP, PPD, CAL) as well as in the visible plaque index after 6 months. This result was paralleled by a significant reduction in the median values of systolic and diastolic 24-hours ambulatory BP, CRP, IL-6, fibrinogen, as well as in the mean values of measures of left ventricular hypertrophy and arterial stiffness [49]. Systolic BP decreased from a median (interquartile range) of 170 (28.8) $\mathrm{mmHg}$ at the time of periodontal treatment to 157.5 (40) $\mathrm{mmHg} 6$ months thereafter. Diastolic BP decreased from a median (interquartile range) of 105 (21.3) $\mathrm{mmHg}$ at the time of periodontal treatment to 95 (11.3) $\mathrm{mmHg} 6$ months thereafter. Left ventricular mass decreased from a mean weight of $234.4 \pm 79.6 \mathrm{~g}$ at baseline to $219.4 \pm 69.2 \mathrm{~g}$ after periodontal treatment. The authors did not explicitly state whether antihypertensive therapy remained stable during follow-up. A full ABPM report, comprehensive of mean $24 \mathrm{~h}$, daily, and nocturnal BP values was not shown. Also, the lack of a control group prevents the full interpretation of the reported findings.

\subsubsection{Effect of Periodontal Therapy on Blood Pressure as a Secondary Outcome}

The effect of periodontal therapy on blood pressure as the secondary outcome in individuals with hypertension has been so far reported by only one study [53]. It was a nonrandomized clinical trial primarily interested in endothelial function that also assessed the effect on BP of nonsurgical periodontal therapy, delivered as needed, in treated hypertensive individuals ( $\mathrm{N}=26$, mean age: $54 \pm 13$ years; $30.8 \%$ women; $34.6 \%$ smokers; mean BMI: $23.2 \pm 3.0$ ) without diabetes mellitus, hyperlipidemia, or CVD who were on a stable ( $\geq 6$ months prior to enrollment) antihypertensive therapy [53]. Hypertension was the only condition they were receiving medications for. No information was available on the BP measurement technique. Periodontal health status was first investigated based on a self-reported questionnaire that assessed the presence of gingival swelling and bleeding, purulent discharge, and tooth mobility and then confirmed by the dentist on a routine oral examination, leading to the identification of 17 (65.4\%) hypertensive individuals who were affected by the disease and needed periodontal treatment. The severity of the disease was not reported. Their office BP, glycolipid profile, and systemic inflammatory markers recorded at baseline and 24 weeks after periodontal treatment were compared with those of a control group of hypertensive individuals without periodontitis $(\mathrm{N}=38$; mean age: $56 \pm 12$ years; $26.3 \%$ women; $42.1 \%$ smokers; mean BMI: $23.1 \pm 2.8$ ). Nonsurgical periodontal treatment included 
subgingival scaling and root planing as needed, with the use of antibiotics for 4-7 days after intensive therapy, and oral hygiene instructions for mouth washing and brushing.

No information was available on the periodontal health markers at baseline or at the 24 week visit.

At baseline, hypertensive individuals with periodontitis had higher serum hs-CRP and IL-6 levels compared with the control group of hypertensive individuals without the disease. At the 24-week final visit, individuals who had received periodontal treatment had significantly lower hsCRP and IL-6 levels compared with their pretreatment values. No other difference in clinical and laboratory features was recorded.

The lack of information on the BP measurement technique, periodontitis markers and periodontal disease severity affect the full interpretation and comparability of the reported findings.

\section{Conclusions}

Periodontitis and hypertension often occur as comorbidities. A positive linear association between the conditions was found, which appears to be driven by the severity of periodontitis and mostly affected the systolic BP component.

In contrast to survey-based research, studies based on BP measurement and oral health status examination found an increased risk of incident hypertension in relation to periodontal disease.

A genetic susceptibility involving inflammation and immunity exists for both hypertension and periodontitis, and polymorphisms in genes involved in the immune function were found to be shared between the two conditions. Environmental factors, like high-salt diet, as well as ecosystemic perturbations contribute to immune cells impairment with effects on periodontal health status and BP.

Based on observational evidence, periodontitis is associated with elevated risk of uncontrolled hypertension despite antihypertensive treatment. Studies on individuals with prehypertension, treated hypertension, or resistant hypertension that examined the effect of periodontal therapy on BP as the primary outcome indicate a parallel improvement in systemic inflammatory markers and BP profile after treatment. Heterogeneity exists in the evidence.

\section{Clinical Implications}

There is a rationale for the assessment of oral health status and BP profile in the presence of either hypertension or periodontitis.
In addition to its proven benefits on BP profile, a low-salt diet might be beneficial for periodontal health status.

The benefits of maintaining or restoring a healthy periodontium might extend to BP profile.

\section{Research Implications}

More mechanistic studies and large randomized clinical trials are warranted.

Acknowledgements We would like to acknowledge the Editors-inChief Prof. Massimo Volpe (High Blood Pressure \& Cardiovascular Prevention) and Prof. Giovanni Lodi (Oral Diseases) and the Journal Publishing Managers Amitabh Prakash (Springer Nature) and Kate Lavallee (Wiley) who arranged the co-publication of this document.

\section{Declarations}

Funding Open access funding provided by Università degli Studi dell'Aquila within the CRUI-CARE Agreement.

Conflict of interest On behalf of all authors, the corresponding authors state that there is no conflict of interest.

Open Access This article is licensed under a Creative Commons Attribution-NonCommercial 4.0 International License, which permits any non-commercial use, sharing, adaptation, distribution and reproduction in any medium or format, as long as you give appropriate credit to the original author(s) and the source, provide a link to the Creative Commons licence, and indicate if changes were made. The images or other third party material in this article are included in the article's Creative Commons licence, unless indicated otherwise in a credit line to the material. If material is not included in the article's Creative Commons licence and your intended use is not permitted by statutory regulation or exceeds the permitted use, you will need to obtain permission directly from the copyright holder. To view a copy of this licence, visit http://creativecommons.org/licenses/by-nc/4.0/.

\section{References}

1. Giampaoli S, Vescio MF, Gaggioli A, Vanuzzo D, [on behalf of the Research Group of the Observatory for Cardiovascular Epidemiology]. Prevalence of Arterial Hypertension in the Italian Population. Istituto Superiore di Sanità. 2021. https://www. epicentro.iss.it/ben/2002/settembre02/2_en. Accessed 1 May 2021.

2. Aimetti M, Perotto S, Castiglione A, Mariani GM, Ferrarotti F, Romano F. Prevalence of periodontitis in an adult population from an urban area in North Italy: findings from a cross-sectional population-based epidemiological survey. J Clin Periodontol. 2015;42:622-31.

3. Loos BG, Van Dyke TE. The role of inflammation and genetics in periodontal disease. Periodontol. 2000;2020(83):26-39.

4. Del Pinto R, Pietropaoli D, Munoz-Aguilera E, D’Aiuto F, Czesnikiewicz-Guzik M, Monaco A, et al. Periodontitis and hypertension: is the association causal? High Blood Press Cardiovasc Prev. 2020;27:281-9. 
5. Czesnikiewicz-Guzik M, Osmenda G, Siedlinski M, Nosalski R, Pelka P, Nowakowski D, et al. Causal association between periodontitis and hypertension: evidence from Mendelian randomization and a randomized controlled trial of non-surgical periodontal therapy. Eur Heart J. 2019;40:3459-70.

6. Sanz M, Marco Del Castillo A, Jepsen S, Gonzalez-Juanatey JR, D'Aiuto F, Bouchard P, et al. Periodontitis and cardiovascular diseases: consensus report. J Clin Periodontol. 2020;47:268-88.

7. Landi L, Grassi G, Sforza NM, Ferri C, Italian working group on Hypertension and Periodontitis (Hy-Per Group). Hypertension and Periodontitis: an Upcoming Joint Report by the Italian Society of Hypertension (SIIA) and the Italian Society of Periodontology and Implantology (SIdP). High Blood Press Cardiovasc Prev. 2021;28:1-3.

8. DiCenso A, Bayley L, Haynes RB. ACP Journal Club. Editorial: Accessing preappraised evidence: fine-tuning the $5 \mathrm{~S}$ model into a 6S model. Ann Intern Med. 2009;151:32-3.

9. Nibali L, Farias BC, Vajgel A, Tu YK, Donos N. Tooth loss in aggressive periodontitis: a systematic review. J Dent Res. 2013;92:868-75.

10. Armitage GC. Development of a classification system for periodontal diseases and conditions. Ann Periodontol. 1999;4:1-6.

11. Page RC, Eke PI. Case definitions for use in population-based surveillance of periodontitis. J Periodontol. 2007;78:1387-99.

12. Preshaw PM. Definitions of periodontal disease in research. J Clin Periodontol. 2009;36:1-2.

13. Tonetti MS, Claffey N, European Workshop in Periodontology group C. Advances in the progression of periodontitis and proposal of definitions of a periodontitis case and disease progression for use in risk factor research. Group $\mathrm{C}$ consensus report of the 5th European Workshop in Periodontology. J Clin Periodontol. 2005;32:210-3.

14. Papapanou PN, Sanz M, Buduneli N, Dietrich T, Feres M, Fine $\mathrm{DH}$, et al. Periodontitis: consensus report of workgroup 2 of the 2017 World Workshop on the Classification of Periodontal and Peri-Implant Diseases and Conditions. J Clin Periodontol. 2018;45(Suppl 20):S162-70.

15. Muñoz Aguilera E, Suvan J, Buti J, Czesnikiewicz-Guzik M, Barbosa Ribeiro A, Orlandi M, et al. Periodontitis is associated with hypertension: a systematic review and meta-analysis. Cardiovasc Res. 2020;116:28-39.

16. Tuominen R, Reunanen A, Paunio M, Paunio I, Aromaa A. Oral health indicators poorly predict coronary heart disease deaths. $\mathrm{J}$ Dent Res. 2003;82:713-8.

17. Martin-Cabezas R, Seelam N, Petit C, Agossa K, Gaertner S, Tenenbaum $\mathrm{H}$, et al. Association between periodontitis and arterial hypertension: a systematic review and meta-analysis. Am Heart J. 2016;180:98-112.

18. Nibali L, Tatarakis N, Needleman I, Tu Y-K, D’Aiuto F, Rizzo M, et al. Clinical review: association between metabolic syndrome and periodontitis: a systematic review and meta-analysis. J Clin Endocrinol Metab. 2013;98:913-20.

19. Tsakos G, Sabbah W, Hingorani AD, Netuveli G, Donos N, Watt $\mathrm{RG}$, et al. Is periodontal inflammation associated with raised blood pressure? Evidence from a National US survey. J Hypertens. 2010;28:2386-93.

20. Pietropaoli D, Del Pinto R, Ferri C, Marzo G, Giannoni M, Ortu $\mathrm{E}$, et al. Association between periodontal inflammation and hypertension using periodontal inflamed surface area and bleeding on probing. J Clin Periodontol. 2020;47:160-72.

21. Whelton PK, Carey RM, Aronow WS, Casey DE Jr, Collins KJ, Dennison Himmelfarb C, et al. 2017 ACC/AHA/AAPA/ABC/ ACPM/AGS/APhA/ASH/ASPC/NMA/PCNA Guideline for the Prevention, Detection, Evaluation, and Management of High Blood Pressure in Adults: Executive Summary: a Report of the American College of Cardiology/American Heart Association
Task Force on Clinical Practice Guidelines. Hypertension. 2018;71:1269-324.

22. Williams B, Mancia G, Spiering W, Agabiti Rosei E, Azizi M, Burnier M, et al. 2018 ESC/ESH Guidelines for the management of arterial hypertension. Eur Heart J. 2018;39:3021-104.

23. Pietropaoli D, Monaco A, D’Aiuto F, Muñoz Aguilera E, Ortu E, Giannoni M, et al. Active gingival inflammation is linked to hypertension. J Hypertens. 2020. https://doi.org/10.1097/HJH. 0000000000002514.

24. Del Pinto R, Pietropaoli D, Monaco A, Giannoni M, Wright JT, Ferri C. Poor oral health negates benefits of antihypertensive treatment: a re-analysis of NHANES data based on $2018 \mathrm{ESC} / \mathrm{ESH}$ hypertension guidelines. J Hypertens. 2019;37:e11.

25. Pietropaoli D, Del Pinto R, Ferri C, Wright JT Jr, Giannoni M, Ortu E, et al. Poor oral health and blood pressure control among US hypertensive adults. Hypertension. 2018;72:1365-73.

26. Rivas-Tumanyan S, Campos M, Zevallos JC, Joshipura KJ. Periodontal disease, hypertension, and blood pressure among older adults in Puerto Rico. J Periodontol. 2013;84:203-11.

27. Eke PI, Page RC, Wei L, Thornton-Evans G, Genco RJ. Update of the case definitions for population-based surveillance of periodontitis. J Periodontol. 2012;83:1449-54.

28. Whelton PK, Carey RM, Aronow WS, Casey DE Jr, Collins KJ, Dennison Himmelfarb C, et al. 2017 ACC/AHA/AAPA/ABC/ ACPM/AGS/APhA/ASH/ASPC/NMA/PCNA guideline for the prevention, detection, evaluation, and management of high blood pressure in adults: a report of the American College of Cardiology/American Heart Association Task Force on Clinical Practice Guidelines. J Am Coll Cardiol. 2018;71:e127-248.

29. Ehret GB, Caulfield MJ. Genes for blood pressure: an opportunity to understand hypertension. Eur Heart J. 2013;34:951-61.

30. Patel RS, Masi S, Taddei S. Understanding the role of genetics in hypertension. Eur Heart J. 2017;38:2309-12.

31. Ehret GB, Ferreira T, Chasman DI, Jackson AU, Schmidt EM, Johnson $\mathrm{T}$, et al. The genetics of blood pressure regulation and its target organs from association studies in 342,415 individuals. Nat Genet. 2016;48:1171-84.

32. Wessel J, Moratorio G, Rao F, Mahata M, Zhang L, Greene W, et al. C-reactive protein, an "intermediate phenotype" for inflammation: human twin studies reveal heritability, association with blood pressure and the metabolic syndrome, and the influence of common polymorphism at catecholaminergic/beta-adrenergic pathway loci. J Hypertens. 2007;25:329-43.

33. Nibali L, Bayliss-Chapman J, Almofareh SA, Zhou Y, Divaris K, Vieira AR. What is the heritability of periodontitis? A systematic review. J Dent Res. 2019;98:632-41.

34. Schaefer AS, Richter GM, Nothnagel M, Manke T, Dommisch $\mathrm{H}$, Jacobs $\mathrm{G}$, et al. A genome-wide association study identifies GLT6D1 as a susceptibility locus for periodontitis. Hum Mol Genet. 2010;19:553-62.

35. Munz M, Richter GM, Loos BG, Jepsen S, Divaris K, Offenbacher $\mathrm{S}$, et al. Meta-analysis of genome-wide association studies of aggressive and chronic periodontitis identifies two novel risk loci. Eur J Hum Genet. 2019;27:102-13.

36. Munz M, Willenborg C, Richter GM, Jockel-Schneider Y, Graetz $\mathrm{C}$, Staufenbiel I, et al. A genome-wide association study identifies nucleotide variants at SIGLEC5 and DEFA1A3 as risk loci for periodontitis. Hum Mol Genet. 2017;26:2577-88.

37. Avril T, Freeman SD, Attrill H, Clarke RG, Crocker PR. Siglec-5 (CD170) can mediate inhibitory signaling in the absence of immunoreceptor tyrosine-based inhibitory motif phosphorylation. J Biol Chem. 2005;280:19843-51.

38. Crocker PR, Paulson JC, Varki A. Siglecs and their roles in the immune system. Nat Rev Immunol. 2007;7:255-66.

39. Evangelou E, Warren HR, Mosen-Ansorena D, Mifsud B, Pazoki R, Gao H, et al. Genetic analysis of over 1 million people 
identifies 535 new loci associated with blood pressure traits. Nat Genet. 2018;50:1412-25.

40. Wu C, Yosef N, Thalhamer T, Zhu C, Xiao S, Kishi Y, et al. Induction of pathogenic TH17 cells by inducible salt-sensing kinase SGK1. Nature. 2013;496:513-7.

41. Jobin K, Stumpf NE, Schwab S, Eichler M, Neubert P, Rauh $\mathrm{M}$, et al. A high-salt diet compromises antibacterial neutrophil responses through hormonal perturbation. Sci Transl Med. 2020. https://doi.org/10.1126/scitranslmed.aay3850.

42. Dutzan N, Kajikawa T, Abusleme L, Greenwell-Wild T, Zuazo CE, Ikeuchi T, et al. A dysbiotic microbiome triggers T17 cells to mediate oral mucosal immunopathology in mice and humans. Sci Transl Med. 2018. https://doi.org/10.1126/scitranslmed.aat0797.

43. Rivas-Tumanyan S, Spiegelman D, Curhan GC, Forman JP, Joshipura KJ. Periodontal disease and incidence of hypertension in the health professionals follow-up study. Am J Hypertens. 2012;25:770-6.

44. Gordon JH, LaMonte MJ, Zhao J, Genco RJ, Cimato TR, Hovey $\mathrm{KM}$, et al. Association of periodontal disease and edentulism with hypertension risk in postmenopausal women. Am J Hypertens. 2019;32:193-201.

45. Kawabata Y, Ekuni D, Miyai H, Kataoka K, Yamane M, Mizutani $\mathrm{S}$, et al. Relationship between prehypertension/hypertension and periodontal disease: a prospective cohort study. Am J Hypertens. 2016;29:388-96.

46. Morita T, Yamazaki Y, Mita A, Takada K, Seto M, Nishinoue N, et al. A cohort study on the association between periodontal disease and the development of metabolic syndrome. J Periodontol. 2010;81:512-9.

47. Sharma S, Sridhar S, McIntosh A, Messow C-M, Aguilera EM, Del Pinto R, et al. Periodontal therapy and treatment of hypertension-alternative to the pharmacological approach. A systematic review and meta-analysis. Pharmacol Res. 2021;166:105511.

48. Zhou Q-B, Xia W-H, Ren J, Yu B-B, Tong X-Z, Chen Y-B, et al. Effect of intensive periodontal therapy on blood pressure and endothelial microparticles in patients with prehypertension and periodontitis: a randomized controlled trial. J Periodontol. 2017;88:711-22.

49. Vidal F, Cordovil I, Figueredo CMS, Fischer RG. Non-surgical periodontal treatment reduces cardiovascular risk in refractory hypertensive patients: a pilot study. J Clin Periodontol. 2013;40:681-7.

50. Chobanian AV, Bakris GL, Black HR, Cushman WC, Green LA, Izzo JL Jr, et al. Seventh report of the joint national committee on prevention, detection, evaluation, and treatment of high blood pressure. Hypertension. 2003;42:1206-52.

51. O'Brien E, Asmar R, Beilin L, Imai Y, Mancia G, Mengden T, et al. Practice guidelines of the European Society of Hypertension for clinic, ambulatory and self blood pressure measurement. J Hypertens. 2005;23:697-701.

52. Mancia G, Fagard R, Narkiewicz K, Redon J, Zanchetti A, Böhm $\mathrm{M}$, et al. $2013 \mathrm{ESH} / \mathrm{ESC}$ guidelines for the management of arterial hypertension: the Task Force for the Management of Arterial Hypertension of the European Society of Hypertension (ESH) and of the European Society of Cardiology (ESC). Eur Heart J. 2013;34:2159-219.

53. Higashi Y, Goto C, Jitsuiki D, Umemura T, Nishioka K, Hidaka T, et al. Periodontal infection is associated with endothelial dysfunction in healthy subjects and hypertensive patients. Hypertension. 2008;51:446-53. 